\title{
Hybrid Position-Force Control of a Cable-Driven Parallel Robot with Experimental Evaluation
}

\author{
W. Kraus, P. Miermeister, V. Schmidt, and A. Pott \\ Fraunhofer Institute for Manufacturing Engineering and Automation IPA in Stuttgart, Germany \\ Correspondence to: W. Kraus (wek@ipa.fhg.de)
}

Received: 1 December 2014 - Revised: 25 May 2015 - Accepted: 8 July 2015 - Published: 4 August 2015

\begin{abstract}
For cable-driven parallel robots elastic cables are used to manipulate a mobile platform in the workspace. In this paper, we present a hybrid position-force control, which allows for applying defined forces on the environment and simultaneous movement along the surface. We propose a synchronous control of the cable forces to ensure the stability of the platform during movement. The performance of the controller is experimentally investigated regarding contact establishment and dynamic behavior during a motion on the cable robot IPAnema 3.
\end{abstract}

\section{Introduction}

Due to their huge workspace, high dynamics and lightweight structure, cable-driven parallel robots, in the following referred to as cable robots, received high interest in the past.

Processes like grinding and polishing demand an accurately controlled contact force to give reliable results. One application example is the surface grinding in the airplane maintenance, which is exemplary setup using oil barrels shown in Fig. 1. Force control also allows compensating for deviations in the work piece geometry by keeping the tool constantly in contact with the surface. There exist solutions like air controlled cylinders or stiffness actuators, which can be installed at the robot's platform to enable for the control of process forces. Osypiuk and Kröger (2011) The drawback is that the system adds additional weight to the platform and the direction of the force is typically fixed w.r.t. the platform frame. Furthermore, these systems make a power and signal supply on the platform necessary.

The aim of the hybrid position-force control is to apply process forces in a programmable direction to a surface while the movement perpendicular to the force vector is position controlled.

The basic approach for the hybrid position-force control was already presented in the early eighties by Raibert and Craig (1981) and its basic structure is shown in Fig. 3. The control problem is divided into parallel control loops for position and force control with their set-points $\boldsymbol{x}_{\text {set }}$ and $\boldsymbol{F}_{\text {set }}$,

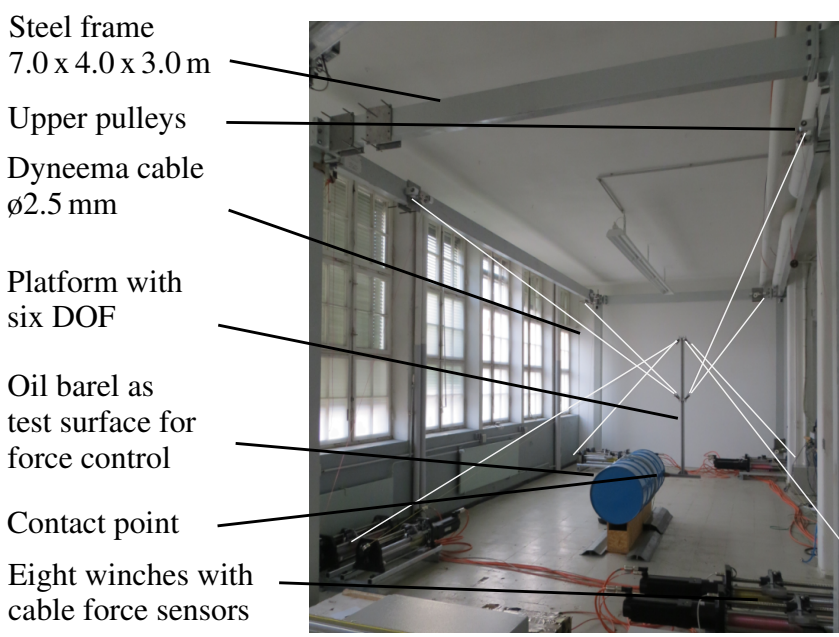

Figure 1. Cable-driven parallel robot IPAnema 3: Robot test set-up.

respectively. With the selection matrix $\mathbf{S}$, the degrees-offreedom (DOF) are assigned to be either force or position controlled. The hybrid control law was implemented already on parallel manipulators in Madani and Moallem (2011) and Deng et al. (2010). For the cable robot, several adaptions are made. The major difference to the classic hybrid control law is the additional cable force control loop to control the internal cable tensions. For a serial robot, the concept of an additional null-space control loop is described in Park (2006). 


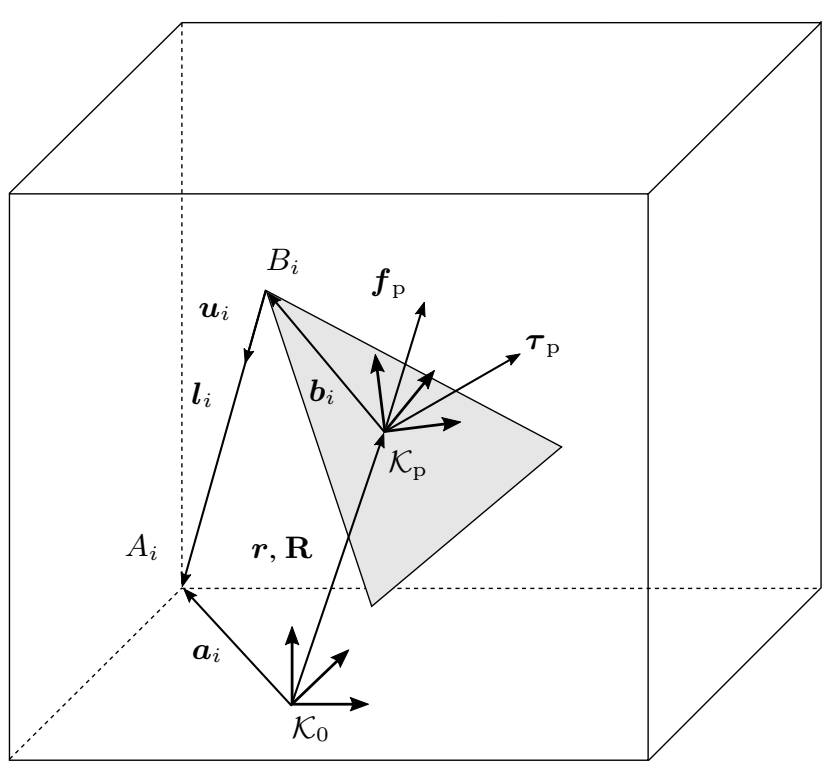

Figure 2. General kinematic parameters.

Another difference is the use of joint space force measurement and transformation into operational space instead of a force-torque sensor at the platform. The cable force sensors are already integrated for cable force control. By using them for wrench measurement, additional costs for sensors can be avoided and no additional sensor has to be added to the platform. We assume a cable robot using closed-loop position control in the joint space, instead of closed-loop position control in operational space. Therefore, no forward kinematics is used which is computationally expensive for a cable robot. The position control is encapsulated in the motion generator. Therefore, the selection matrix $\mathbf{S}$ cannot be realized for position control and the decoupling is realized in the force control implementation.

Beside the hybrid position-force control, the contact establishment is challenging. The major concern regarding stability are the transient system properties when the platform comes in contact with the environment. The contact problem was investigated for both serial (Albu-Schaeffer et al., 2003; Raibert and Craig, 1981), and classical parallel robots Tang et al. (2012). In Assuncao and Schumacher (2003) this problem is solved by a state machine based approach using acceleration and brake controllers for bringing the robot into contact. An alternative approach for eliminating the switching strategy is presented in Almeida et al. (1999) using an adaptive gain of the impedance controller. In Reisinger et al. (2011) Moreau's sweeping process is applied for the transition during contact establishment.

In this paper, we present the design and experimental evaluation of a operational space force control for a cable robot.

In addition to the known contact problem, we have to ensure that the cables remain under tension.

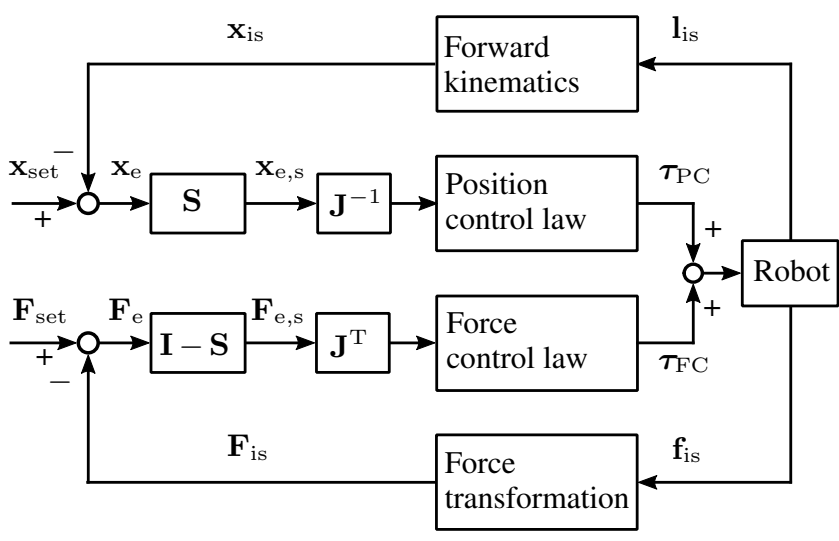

Figure 3. Hybrid position and force control proposed by Raibert and Craig (1981).

The state machine based approach presented in Assuncao and Schumacher (2003) is adapted for the cable robot. To keep the cables under tension, we introduce a decoupled cable force controller to tension the cables during the contact force control. For this, the cable force control presented in Kraus et al. (2014) is modified regarding the set-point determination. A task coordinate system is established to realize the hybrid position-force control. For motion control, the trajectory planning and interpolation is done in operational space and transformed with the inverse kinematics to position set-points in joint space. This structure is typically used in commercial CNC controllers. We chose position-controlled drives and not a torque-based approach to make use of stateof-the art industrial servo drives and the robustness of the decentralized position control. To program the desired force vector with standard industrial machine interfaces, new commands based on standardized G-Code are established.

This paper is organized as follows: The robot model including kinematic and the determination of cable force distributions are summarized in Sect. 2. The design and implementation of the operational space force controller is described in Sect. 3. Experimental results are presented and discussed in Sect. 4. Finally, conclusions and an outlook on future work are given in Sect. 5.

\section{Kinematic and static model}

For completeness, we briefly review the kinematic model for a spatial robot with $n=6$ DOF. Verhoeven (2004) The geometry of the robot is described by the proximal anchor points on the robot base $\boldsymbol{a}_{i}$ and the distal anchor points on the platform $\boldsymbol{b}_{i}$ as shown in Fig. 2. The index $i$ denotes the cable number and $m$ is the absolute number of cables. By applying a vector loop, the cable vector $\boldsymbol{l}_{i}$ follows as

$\boldsymbol{l}_{i}=\boldsymbol{a}_{i}-\boldsymbol{r}-\mathbf{R} \boldsymbol{b}_{i}$, 
while $\boldsymbol{r}$ is the platform position vector and rotation matrix $\mathbf{R}$ describes the orientation of the mobile platform frame $\mathcal{K}_{\mathrm{p}}$ w.r.t. the base coordinate frame $\mathcal{K}_{0}$.

The structure equation with $\mathcal{K}_{0}$ the structure matrix $\mathbf{A}^{\mathrm{T}}$ describes the force and torque equilibrium at the platform for a given cable force distribution $\boldsymbol{f}$ and can be written as

$$
\underbrace{\left[\begin{array}{ccc}
\boldsymbol{u}_{1} & \cdots & \boldsymbol{u}_{m} \\
\boldsymbol{b}_{1} \times \boldsymbol{u}_{1} & \cdots & \boldsymbol{b}_{m} \times \mathbf{u}_{m}
\end{array}\right]}_{\mathbf{A}^{\mathrm{T}}(\boldsymbol{r}, \mathbf{R})} \underbrace{\left[\begin{array}{c}
f_{1} \\
\vdots \\
f_{m}
\end{array}\right]}_{\boldsymbol{f}}=-\underbrace{\left[\begin{array}{c}
\boldsymbol{f}_{\mathrm{p}} \\
\boldsymbol{\tau}_{\mathrm{p}}
\end{array}\right]}_{\boldsymbol{w}},
$$

with $\boldsymbol{u}_{i}=\frac{\boldsymbol{l}_{i}}{\left\|\boldsymbol{l}_{i}\right\|}$ being the unit vector of the cables directing from the mobile platform to the base. The applied wrench $\boldsymbol{w}$ consists of external forces $\boldsymbol{f}_{\mathrm{p}}$ and torques $\boldsymbol{\tau}_{\mathrm{p}}$ and also includes the weight $m_{\mathrm{p}}$ of the platform.

For the cable force control, a force distribution $f$ has to be calculated, which solves the structure Eq. (2) and is continuous along a trajectory. There exist several approaches which fulfill these requirements like Lamaury and Gouttefarde (2012) or Mikelsons et al. (2008). In this paper, the advanced closed-form solution presented in Pott (2013) is applied. It is based on the closed-form solution for the structure matrix presented in Pott et al. (2009). In this approach, a reference cable force $f_{\text {ref }}=\left(f_{\text {min }}+f_{\text {max }}\right) / 2$ is introduced which is based on the minimum cable force $f_{\min }$ and maximum cable force $f_{\text {max }}$, respectively. With the MoorePenrose matrix inverse $\mathbf{A}^{+\mathrm{T}}$, the least square optimal force distribution is obtained by

$\boldsymbol{f}=\boldsymbol{f}_{\text {ref }}-\mathbf{A}^{+\mathrm{T}}\left(\boldsymbol{w}+\mathbf{A}^{\mathrm{T}} \boldsymbol{f}_{\text {ref }}\right)$.

The redundancy $r=m-n$ defines the rank of the null-space of the structure matrix. For the IPAnema cable robot with $r=2$, the workspace derived with the closed-form solution amounts only $57.5 \%$ of the possible workspace. This motivated for the further development and ended in the advanced closed-form method. The algorithm and its application to workspace analysis of cable robots was firstly presented in Pott (2013).

The advanced closed-form algorithm consists of the evaluation of the closed-form solution and an order reduction of the linear equation system in the case, the cable bounds are violated. The largest violation of the cable bounds is detected and the cable force is set to the corresponding limit value. Next, this cable is excluded from structure matrix and the exerted force and torque of this cable is added to the wrench. This procedure is repeated until a feasible solution is reached or the structure matrix is quadratic and no further reduction is possible. The computational effort is strictly bounded. In the worst case, $r$ iterations have to be performed. The description of the robot model is hereby completed and next the controller design for hybrid position-force control is presented.

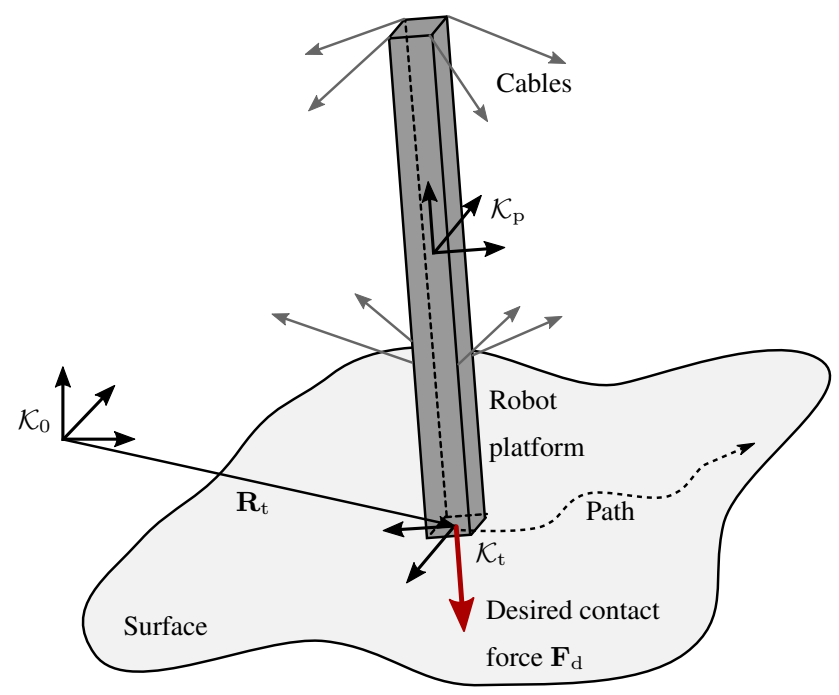

Figure 4. Principle set-up at the mobile platform and coordinate systems for hybrid position-force control.

\section{Controller design and implementation}

\subsection{Controller design}

The operational space force control aims at the control of the wrench $\boldsymbol{w}$ in a given direction e.g. perpendicular to the surface of the environment. While using a cable robot for applying the contact force, additionally the cables have to be kept under tension. Especially for the simultaneous position controlled movement in the tangential plane this is important, as friction forces have to be overcome. To realize the hybrid position-force control for a cable robot, the control problem is divided into three parts:

- The operational space position control gives a desired position in operational space $\boldsymbol{x}_{\text {set,PC }}$ to move the platform along a path.

- The operational space force control generates an offset in the operational space position $\boldsymbol{x}_{\text {set,OFC }}$ allowing to control the external platform wrench i.e. contact force using joint space position control.

- The cable force control for keeping the cables under tension. For this, a feasible force distribution is calculated for the measured wrench. The controller output is the modification $\boldsymbol{l}_{\mathrm{CFC}}$ of the cable length set-point.

With the inverse kinematics (IK) the output of the three controllers are summed up by

$\boldsymbol{l}_{\mathrm{set}}=\mathrm{IK}\left\{\boldsymbol{x}_{\mathrm{set}, \mathrm{PC}}+\boldsymbol{x}_{\mathrm{set}, \mathrm{OFC}}\right\}+\boldsymbol{l}_{\mathrm{CFC}}$,

to the cable length set-point $\boldsymbol{l}_{\text {set }}$ which is commanded to the servo drives. The control structure is visualized in Fig. 5. In the following, the operational space and cable force control are described in detail. 


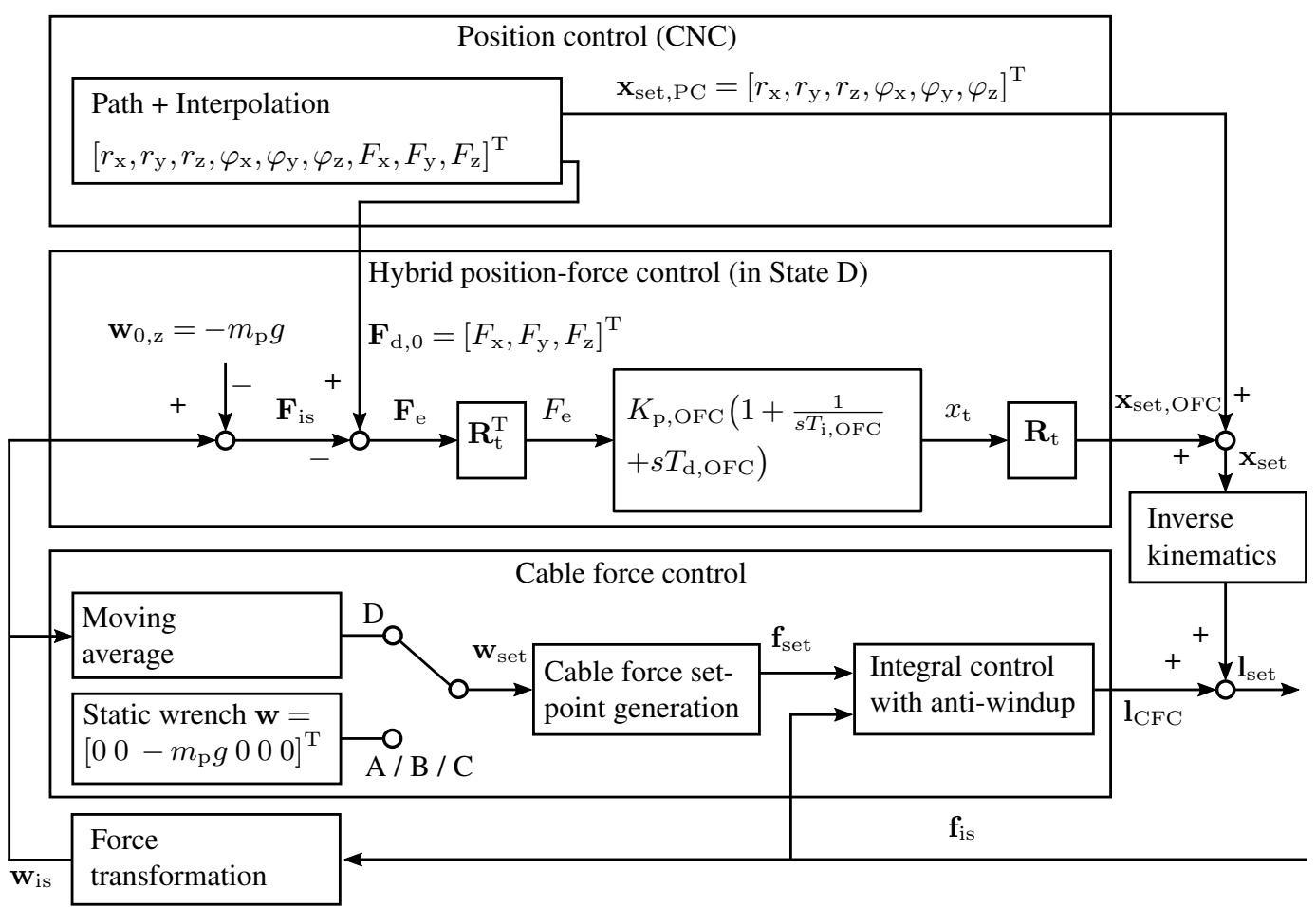

Figure 5. Proposed implementation of the hybrid position-force control with cable force control.

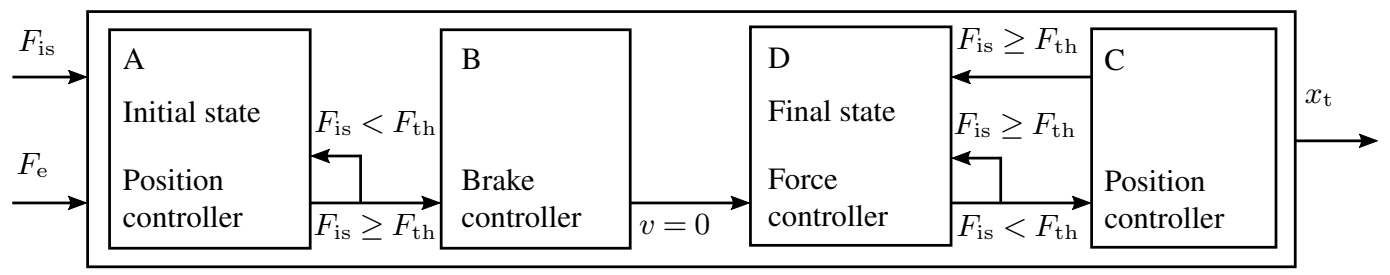

Figure 6. State machine with transition conditions for the contact establishment.

\subsection{Operational Space Force Control}

The main challenge of the operational space force control is the contact establishment. When the platform comes in contact, the system properties change abruptly. We adapt the approach presented by Assuncao and Schumacher (2003) which uses a state machine. Basically, the contact is established in position control (States A-C) and closed-loop force control is performed when the platform is in contact. The contact establishment is structured into four states, as illustrated in Fig. 6. The inputs of the state machine are the actual contact force $F_{\text {is }}$ and the deviation from the desired contact force $F_{\mathrm{e}}=F_{\mathrm{set}}-F_{\text {is }}$. The output is the position offset $x_{\mathrm{t}}$ in the direction of the desired force vector.

In state $\mathrm{A}$, the platform is moved in the direction of the surface with a maximal velocity $v_{\max }$. During the movement, the wrench is observed. The contact between the platform and the surface is detected when the threshold force $F_{\text {th }}$ is exceeded. As the measured wrench is disturbed by measure- ment errors, we also observe the derivative of the force to identify the contact. When the contact is detected, the platform is slowed down till stand still in state B. Next in state D, the closed-loop force control is activated as shown in Fig. 5. A PID controller with the transfer function

$G_{\mathrm{c}}(s)=\frac{\mathcal{L}\left\{x_{\mathrm{t}}(t)\right\}}{\mathcal{L}\left\{F_{\mathrm{e}}(t)\right\}}=K_{\mathrm{p}, \mathrm{OFC}}\left(1+\frac{1}{s T_{\mathrm{i}, \mathrm{OFC}}}+s T_{\mathrm{d}, \mathrm{OFC}}\right)$,

is applied, where $K_{\mathrm{p}, \text { OFC }}$ denotes the gain, $T_{\mathrm{i}, \text { OFC }}$ the integrator reset time and $T_{\mathrm{d}, \mathrm{OFC}}$ the derivative time. The control parameters are determined experimentally and presented in Table 1. When the robot loses the contact to the surface, this means the force is less than the threshold force, the state machine switches to state $\mathrm{C}$ and a position controller brings the platform again in contact.

The operational space force control is based on an indirect force measurement using the cable force sensors. For the transformation from joint to operational space, the structure 


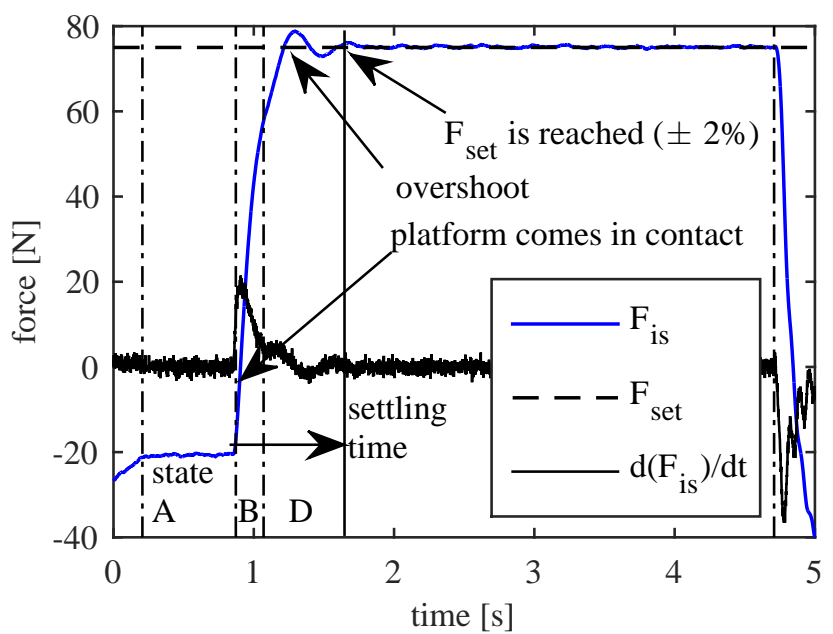

Figure 7. Progression of the force during contact establishment, force control and release.

matrix $\mathbf{A}^{\mathrm{T}}$, describing the actual cable directions, is used. The estimated wrench $\boldsymbol{w}_{\text {is }}$ is derived by

$\boldsymbol{w}_{\text {is }}=-\mathbf{A}^{\mathrm{T}} \boldsymbol{f}_{\text {is }}$,

where $f_{\text {is }}$ denotes the measured cable forces. The actual force vector w.r.t. the world frame $\boldsymbol{F}_{\text {is }}$ is derived from the actual wrench under consideration of the own-weight of the platform $m_{\mathrm{p}} g$.

We introduce a task coordinate system $\mathcal{K}_{\mathrm{t}}$ as visualized in Fig. 4, which lies in the contact point and the $z$ axis coincides with the desired force direction. The transformation from world $\mathcal{K}_{0}$ to task coordinate system $\mathcal{K}_{\mathrm{t}}$ is described by the rotation matrix $\mathbf{R}_{\mathrm{t}}$. The desired force vector $\boldsymbol{F}_{\text {set }, 0}$ is transformed by

$\boldsymbol{F}_{\text {set }, t}=\mathbf{R}_{\mathrm{t}}^{\mathrm{T}} \boldsymbol{F}_{\text {set }, 0}$, with $\boldsymbol{F}_{\text {set }, 0}=\left[\begin{array}{lll}0 & 0 & \boldsymbol{F}_{\text {set }}\end{array}\right]^{\mathrm{T}}$,

to the task coordinate system, where the $z$ component represents the scalar desired force $\boldsymbol{F}_{\text {set }}$. With the coordinate transformation, the force control can be designed as a single input single output controller.

\subsection{Cable force control}

Redundant cable robots have the property that the cables can be tensed against each other without exerting an external force. In the present control architecture, the external forces are controlled by the operational space force control. Therefore, the cable force control aims at the control of the internal tensions and has to be decoupled from the operational space force control. As the wrench is controlled by the operational space force control, we propose to use the measured wrench $\boldsymbol{w}_{\text {is }}$ for the determination of the set-point of the cable forces $f_{\text {set }}$ according to (Eq. 3). This ensures that the controlled cable force is compatible to the actual external forces and does
Table 1. Control parameters of hybrid position-force control (controller is experimentally tuned).

\begin{tabular}{llll}
\hline parameter & definition & value & unit \\
\hline$T_{\mathrm{MA}}$ & $\begin{array}{l}\text { time constant of moving } \\
\text { average for wrench }\end{array}$ & 2.0 & $\mathrm{~s}$ \\
& $\begin{array}{l}\text { filtering } \\
\text { integrator reset time }\end{array}$ & 2.6 & $\mathrm{~s}$ \\
$T_{\mathrm{i}, \mathrm{OFC}}$ & derivative time constant & 0.03 & $\mathrm{~s}^{-1}$ \\
$T_{\mathrm{d}, \mathrm{OFC}}$ & proportional gain & $22000^{-1}$ & $\mathrm{~m} \mathrm{~N}^{-1}$ \\
$K_{\mathrm{p}, \mathrm{OFC}}$ & (compliance) & & \\
& minimum cable force & 100 & $\mathrm{~N}$ \\
$f_{\min }$ & maximum cable force & 1000 & $\mathrm{~N}$ \\
$f_{\max }$ & threshold force & 25 & $\mathrm{~N}$ \\
$F_{\text {th }}$ & maximum approaching & 0.05 & $\mathrm{~m} \mathrm{~s}^{-1}$ \\
$v_{\max }$ & velocity & & \\
& & &
\end{tabular}

not influence the motion of the platform. To avoid instabilities due to the synchronous control of the external wrench and the internal cable forces, a moving average is applied on the wrench to smooth the measurement signal (low pass filter) and, thus, increase the stability margins of the control loop. The control error in cable force $f_{\mathrm{e}}$ is established by

$f_{\text {e }}=f_{\text {set }}-f_{\text {is }}$.

The integral control with anti-windup is realized according to Kraus et al. (2014).

During the contact establishment, the system properties and also the wrench change abruptly. This leads to the destabilization of the contact controller. In this transient phase, the cable force controller also influences the external forces and additionally disturbs the contact controller. To avoid this problem, the cable force control assumes the gravitational force $m_{\mathrm{p}} g$ as wrench during contact establishment which corresponds to the states $\mathrm{A}$ to $\mathrm{C}$ of the state machine. With this approach, we can eliminate additional sources of instabilities due to the internal cable force control. After contact establishment, the cable force control is enabled again.

\subsection{Implementation}

The CNC executes the position control for a path programmed in G-code. Three additional axes were added to the $\mathrm{CNC}$, which are interpreted as desired force vector. These axes were handled as movement axes and were interpolated in the interpolation cycle. In this way, the force vector changes smoothly and is consistent with the movement commands along the surface.

\section{Experimental evaluation}

\subsection{Test platform}

For the experimental evaluation the control algorithms are implemented on the cable robot IPAnema 3 using eight ca- 
Table 2. Experimental evaluation of the contact establishment at different positions and resulting overshoot and settling time.

\begin{tabular}{lrrrr}
\hline position & \multicolumn{2}{c}{ overshoot } & \multicolumn{2}{c}{ settling time } \\
& mean [\%] & $\sigma[\%]$ & mean [s] & $\sigma[\mathrm{s}]$ \\
\hline 1 & 9.357 & 1.171 & 0.781 & 0.191 \\
2 & 8.698 & 0.840 & 1.378 & 0.242 \\
3 & 5.692 & 0.351 & 0.981 & 0.093 \\
4 & 4.299 & 0.927 & 0.866 & 0.138 \\
5 & 8.573 & 0.486 & 0.977 & 0.081 \\
6 & 8.329 & 0.461 & 1.035 & 0.019 \\
7 & 18.106 & 2.227 & 0.804 & 0.156 \\
8 & 15.024 & 2.857 & 0.742 & 0.209 \\
9 & 5.100 & 0.353 & 0.889 & 0.010 \\
10 & 3.910 & 0.067 & 3.517 & 0.413 \\
\hline average & 8.709 & - & 1.197 & - \\
\hline
\end{tabular}

bles and a straight line platform as shown in Fig. 1. The robot frame measures $7 \mathrm{~m}$ by $4 \mathrm{~m}$ and is $3 \mathrm{~m}$ high.

The robot control is realized on an industrial PC with Beckhoff TwinCAT $3.1 \mathrm{CNC}$ at a cycle time of $1 \mathrm{~ms}$. The field bus protocol is EtherCAT. For the force measurement each winch is equipped with a cable force sensor of type Tecsis F2301 with a measurement range up to $4000 \mathrm{~N}$. The analog output signal of the force sensors is digitized in A/Dconverters and sent via the field bus to the control. The cable type is LIROS D-Pro 01505-0250 based on Dyneema SK 75 fiber (Polyethylene) with a diameter of $2.50 \mathrm{~mm}$.

\subsection{Contact establishment}

For the investigation of the contact establishment, a vertical force on the floor is applied. The resulting progression of the force during force control is shown in Fig. 7. The time derivative of the contact force $\mathrm{d}\left(\mathrm{F}_{\mathrm{is}}\right) / \mathrm{d} t$ is noisy but clearly signals when the platform comes in contact.

One interesting point is the contact establishment under the condition of changing robot stiffness in the contact. For this, the operational space force control was applied on the floor at ten different positions which are visualized in Fig. 8. Position 1 lies in the center of the workspace, whereas 7 and 9 are on the edge of robot's footprint.

The evaluation criteria for the contact establishment are the overshoot and settling time. For the experiment the approaching velocity is $50 \mathrm{~mm} \mathrm{~s}^{-1}$ and the desired force amounts to $75 \mathrm{~N}$. The experimental results are presented in Table 2. We can observe a maximum overshoot of $18.2 \%$ whereas the average lies at $8.7 \%$. The settling time is defined as the time from the first contact to the surface until the control error is smaller than $2 \%$. The mean settling time is in average $1.2 \mathrm{~s}$.

The results show a compromise between an acceptable overshoot and a settling time. By changing the approaching

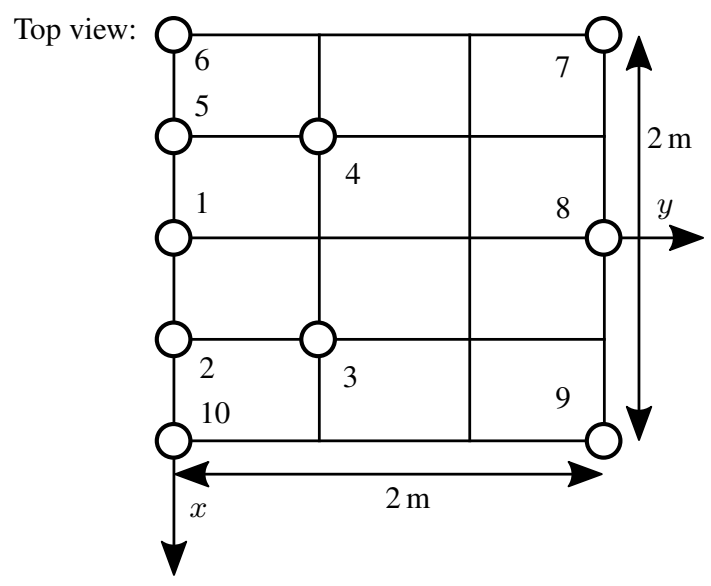

Figure 8. Experimental evaluation of the contact establishment at different positions.

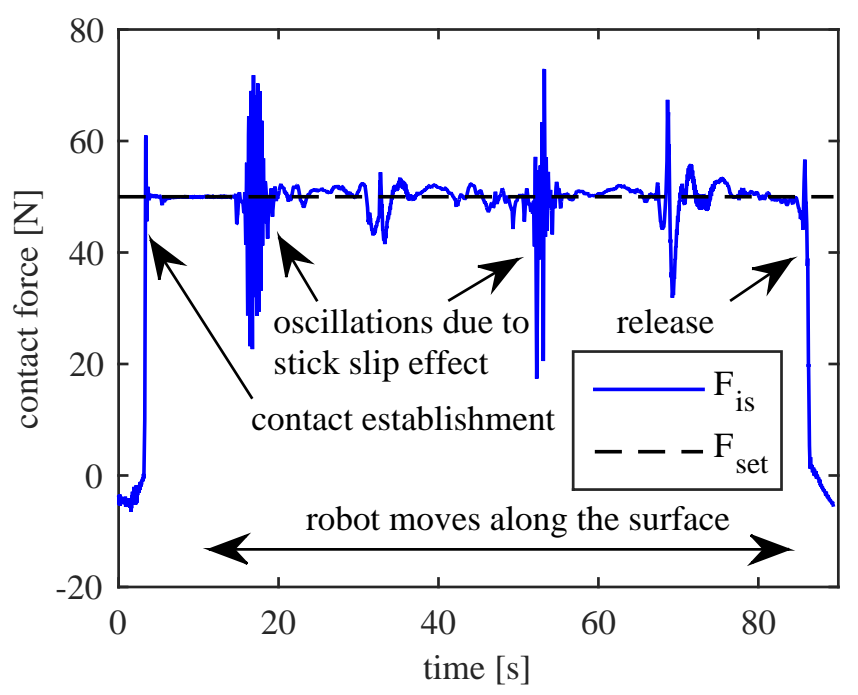

Figure 9. Progression of the force normal to the surface on the oil barrel.

velocity, this compromise can be influenced in favor of one evaluation criteria.

The settling time lies in a range of experimental results which can be found in literature. In Kroeger et al. (2004) the settling time for a serial industrial manipulator lies between 0.6 and $1.7 \mathrm{~s}$. In another scenario shown in Lange et al. (2013) a settling time of $0.8 \mathrm{~s}$ for an industrial robot was achieved.

\subsection{Following behavior}

The following behavior of the force control during a simultaneous motion of the platform was investigated on oil barrels.

The test set-up is shown in Fig. 1. The test scenario is exemplary for a spherical surface which can be found at wings of airplanes or blades of wind turbines. The path along the 
oil barrel is programmed offline. In addition, we also programmed the desired force vector which is aligned to the normal vector of the surface.

The quantitative behavior of the contact force along the surface is shown in Fig. 9. The desired force is $50 \mathrm{~N}$. As the contact point of the platform to the oil barrel is eccentric, the stiffness is low and stick slip effects in the contact generate oscillations. The oscillation is relatively high, when the robot moves position controlled into a direction where it is not very stiff. Since the cables are tensed, these critical positions were overcome. For the position and orientation measurement during the trajectory no measurement equipment was available. Qualitatively, the platform followed well the trajectory.

\section{Conclusions}

In this paper, we proposed an adaption of the well-known hybrid position-force control for a cable robot. By using the measured wrench as input for the set-point determination of cable forces we were able to tense the cables during the contact control. The experimental evaluations on the IPAnema 3 are promising. Further investigation could analyze a specific process by attaching a tool and running the process and analyzing how the controller performs when including additional noise associated with running the process. Another interesting point is the online identification of the stiffness in the contact and how such knowledge can improve the control quality.

Acknowledgements. This work was supported by the FhG Internal Programs under Grant no. WISA 823244.

Edited by: J. Schmiedeler

Reviewed by: S. Y. Ko and one anonymous referee

\section{References}

Albu-Schaeffer, A., Ott, C., Frese, U., and Hirzinger, G.: Cartesian Impedance Control of Redundant Robots: Recent Results with the DLR-Light-Weight-Arms, IEEE International Conference on Robotics and Automation, 2003.

Almeida, F., Lopes, A., and Abreu, P.: Force-Impedance Control a new control strategy of robotic manipulators, Recent Advances in Mechatronics, 1999.

Assuncao, V. and Schumacher, W.: Hybrid Force Control for Parallel Manipulators, 11th Mediterranean Conference on Control and Automation (MED), Rhodos, Griechenland, 2003.
Deng, W., Lee, H. J., and Jeh-Won, L.: Dynamic Hybrid PositionForce Control for Parallel Robot Manipulators, ROMANSY 18 Robot Design, Dynamics and Control, 2010.

Kraus, W., Schmidt, V., Rajendra, P., and Pott, A.: System Identification and Cable Force Control for a Cable-Driven Parallel Robot with Industrial Servo Drives, IEEE International Conference on Robotics and Automation, 2014.

Kroeger, T., Finkemeyer, B., Heuck, M., and Wahl, F. M.: Adaptive implicit hybrid force/pose control of industrial manipulators: compliant motion experiments, IEEE/RSJ International Conference on Intelligent Robots and Systems (IROS), 2004.

Lamaury, J. and Gouttefarde, M.: A Tension Distribution Method with Improved Computational Efficiency, in: Cable-Driven Parallel Robots, Springer, 71-85, 2012.

Lange, F., Bertleff, W., and Suppa, M.: Force and trajectory control of industrial robots in stiff contact, IEEE International Conference on Robotics and Automation, 2013.

Madani, M. and Moallem, M.: Hybrid position/force control of a flexible parallel manipulator, Journal of the Franklin Institute, 999-1012, 2011.

Mikelsons, L., Bruckmann, T., Schramm, D., and Hiller, M.: A Real-Time Capable Force Calculation Algorithm for Redundant Tendon-Based Parallel Manipulators, in: ICRA, Pasadena, 2008.

Osypiuk, R. and Kröger, T.: Parallel Stiffness Actuators with Six Degrees of Freedom for Efficient Force/Torque Control Applications, in: Robotic Systems for Handling and Assembly, Springer, vol. 67, 2011.

Park, J.: Control strategies for robots in contact, Ph.D. thesis, Stanford University, 2006.

Pott, A.: An improved Force Distribution Algorithm for OverConstrained Cable-Driven Parallel Robots, in: Computational Kinematics, Springer, 2013.

Pott, A., Bruckmann, T., and Mikelsons, L.: Closed-form Force Distribution for Parallel Wire Robots, in: Computational Kinematics, Springer, 25-34, 2009.

Raibert, M. H. and Craig, J. J.: Hybrid Position/Force Control of Manipulators, in: Journal of Dynamic Systems, Measurement, and Control, vol. 103, 1981.

Reisinger, T., Wobbe, F., Kolbus, M., and Schumacher, W.: Integrated Force and Motion Control of Parallel Robots - Part 2: Constrained Space, in: Robotic Systems for Handling and Assembly, Springer, 2011.

Tang, H., Yao, J., Chengz, L., and Zhao, Y.: Hybrid Position Force Control Investigation of parallel machine tool with redundant action, Applied Mechanics and Materials, 2012.

Verhoeven, R.: Analysis of the Workspace of Tendon-based Stewart Platforms, Ph.D. thesis, University of Duisburg-Essen, Duisburg, 2004. 